\title{
Short Cycles in Digraphs with Local Average Outdegree at Least Two
}

\author{
Jian Shen \\ Department of Mathematics \\ Southwest Texas State University \\ San Marcos, TX 78666 \\ js48@swt.edu
}

Submitted: May 29, 2001; Accepted: Jun 17, 2003; Published: Jun 27, 2003

MR Subject Classifications: 05C20, 05C35, 05C38

\begin{abstract}
Suppose $G$ is a strongly connected digraph with order $n$ girth $g$ and diameter $d$. We prove that $d+g \leq n$ if $G$ contains no $\operatorname{arcs}(u, v)$ with $\operatorname{deg}^{+}(u)=1$ and $\operatorname{deg}^{+}(v) \leq 2$.

Caccetta and Häggkvist showed in 1978 that any digraph of order $n$ with minimum outdegree 2 contains a cycle of length at most $\lceil n / 2\rceil$. Applying the abovementioned result, we improve their result by replacing the minimum outdegree condition by some weaker conditions involving the local average outdegree. In particular, we prove that, for any digraph $G$ of order $n$, if either

1. $G$ has minimum outdegree 1 and $\operatorname{deg}^{+}(u)+\operatorname{deg}^{+}(v) \geq 4$ for all arcs $(u, v)$, or

2. $\operatorname{deg}^{+}(u)+\operatorname{deg}^{+}(v) \geq 3$ for all pairs of distinct vertices $u, v$, then $G$ contains a cycle of length at most $\lceil n / 2\rceil$.
\end{abstract}

\section{Introduction}

Let $G=(V, E)$ denote a digraph on $n=n(G)$ vertices. Loops are permitted but no multiple arcs. All cycles considered here are directed cycles. If $G$ has at least one cycle, the minimum length of a cycle in $G$ is called the girth of $G$, denoted $g(G)$. On the other hand, if $G$ contains no cycles, the girth of $G$ is defined to be infinity. The number of arcs leaving (resp. entering) a vertex $u$ is called the outdegree (resp. indegree) of $u$, denoted $\operatorname{deg}^{+}(u)$ (resp. $\operatorname{deg}^{-}(u)$ ). A digraph $G$ is said to be $r$-regular if the outdegree and indegree of each vertex are both exactly $r$. The notation $\delta^{+}(G)$ is used to denote the minimum 
outdegree of $G$. Suppose $u, v$ are two vertices in a strongly connected digraph $G$. The distance from $u$ to $v$ is the length of a shortest path from $u$ to $v$ in $G$. The diameter of $G$, denoted $d(G)$, is the maximum distance among all ordered pairs of vertices in $G$.

In 1970, Behzad [1] proved that the girth of any 2-regular digraph of order $n$ is at most $\lceil n / 2\rceil$. This bound is best possible in the sense that the girth of the Cayley digraph $\operatorname{Cay}\left(\mathbf{Z}_{n},\{1,2\}\right)$ on a cyclic group $\left(\mathbf{Z}_{n},+\right)$ of order $n$ is $\lceil n / 2\rceil$. The regularity condition can be relaxed however. For example, Caccetta and Häggkvist [4] made the following improvement in 1978.

Lemma 1 The girth of any digraph of order $n$ with $\delta^{+}(G) \geq 2$ is at most $\lceil n / 2\rceil$.

It is easy to see that any digraph $G$ with $\delta^{+}(G) \geq 2$ has a spanning subdigraph whose each vertex has outdegree exactly 2 . Since the girth of $G$ is no larger than that of any of its subdigraphs, the following lemma is equivalent to Lemma 1.

Lemma 1' Suppose $G$ is a digraph of order $n$ with girth $g$. If $\operatorname{deg}^{+}(u)=2$ for each vertex $u$ in $G$, then $g \leq\lceil n / 2\rceil$.

It is noted that Lemma 1 is a special case of the following well-known conjecture of Caccetta and Häggkvist [4].

Conjecture 1 Let $G$ be a digraph of order $n$ with girth $g$ and $\delta^{+}(G) \geq r$. Then $g \leq\lceil n / r\rceil$.

The Caccetta-Häggkvist conjecture has been proved for $r \leq 5$ by the work of various authors [4], [6], [7]. Recently, the author showed that the conjecture holds when either $n \geq 2 r^{2}-3 r+1[10]$ or $n \leq r(3+\sqrt{7}) / 2$ [9]. While the general conjecture is still open, it is worth mentioning the following result of Chvátal and Szemerédi [5].

Lemma 2 Let $G$ be a digraph of order $n$ with $\delta^{+}(G) \geq r$. Then $g \leq n / r+2500$.

In 1988, Nishimura [8] refined the proof of Chvátal and Szemerédi, reducing the additive constant in Lemma 2 from 2500 to 304. Recently, the author further reduced the additive constant from 304 to 73 [11]. We mention here that Conjecture 1 is stronger than a similar conjecture of Behzad, Chartrand and Wall [2] in which the digraphs are assumed to be regular. For more details, we refer to [3] and [10].

Recently, the following extension of Lemma 1 was obtained in [10] by considering the number of vertices in $G$ with outdegree exactly 1 . 
Lemma 3 Suppose $G$ is a digraph of order $n$ with girth $g$ and $\delta^{+}(G) \geq 1$. Let $t_{1}$ be the number of vertices having outdegree exactly 1 in $G$. Then

$$
g \leq \begin{cases}\lceil n / 2\rceil & \text { if } t_{1}=0 \\ \left\lceil\left(n+t_{1}-1\right) / 2\right\rceil & \text { if } t_{1} \geq 1\end{cases}
$$

The motivation of this paper is to improve Lemma 1 by replacing the condition $\delta^{+}(G) \geq 2$ by some weaker ones involving the local average outdegree. We begin with an example below showing that a global average outdegree of at least 2 in a strongly connected digraph of order $n$ does not guarantee the existence of short cycles (cycles of length at most $\lceil n / 2\rceil$ ). Indeed the girth of such a digraph may be as large as $n-\sqrt{2 n}$.

Example 1. Let $n \geq 7$ and $r=[\sqrt{2 n}]+1$. Then $r \leq n-2$. Let $G=(V, E)$, where $V=\{i: 0 \leq i \leq n-1\}$ and $E=\{(0, n-1)\} \cup\{(i+1, i): r \leq i \leq n-2\} \cup\{(i, j): 0 \leq$ $j<i \leq r\}$. Then the global average outdegree of $G$ is

$$
\frac{1}{n} \sum_{i=0}^{n-1} \operatorname{deg}^{+}(i)=\frac{1}{n}\left(n-r+\sum_{i=1}^{r} i\right)=1+r(r-1) /(2 n)>2
$$

and the girth of $G$ is $n-r+1=n-\lceil\sqrt{2 n}\rceil$.

Although the global average outdegree in Example 1 is larger than 2, the average outdegree of the vertices $i, 1 \leq i \leq r$, is as large as $(r+1) / 2$, while the average outdegree of the vertices $i, r+1 \leq i \leq n-1$, is as small as 1 . This unbalanced distribution of outdegrees among all vertices in $G$ makes the girth of $G$ very large. Thus in order to improve Lemma 1, one needs to consider certain local average outdegree conditions. In particular, we consider in this paper the sums of outdegrees of each pair of adjacent vertices in $G$.

An arc $(u, v) \in E$ is called special if $\operatorname{deg}^{+}(u)=1$ and $\operatorname{deg}^{+}(v) \leq 2$. Thus, in any digraph, the number of special arcs is at most the number of vertices with outdegree exactly 1. Suppose $G$ is a strongly connected digraph with global average outdegree 2 . Since the local average outdegree at each special arc is less than 2, the number of special arcs in $G$ may be considered as a rough measure of how balanced the distribution of the outdegrees of $G$ is. Thus one may expect that the number of special arcs in $G$ has some effect on the girth of $G$. Indeed, we suspect that the greater the number of special arcs in $G$, the larger the possible girth of $G$ may be.

In this paper, we first prove the following relationship,

$$
d \leq n-g+t,
$$

among the diameter $d$, the order $n$, the girth $g$, and the number $t$ of special arcs of any strongly connected digraph. By using this result, we improve Lemma 3 by showing that, 
for any digraph with $\delta^{+}(G) \geq 1$,

$$
g \leq \begin{cases}\left\lceil\frac{n}{2}\right\rceil & \text { if } t=0 \\ \left\lceil\frac{n+t-1}{2}\right\rceil & \text { if } t \geq 1\end{cases}
$$

In particular, if $\delta^{+}(G) \geq 1$ and $\operatorname{deg}^{+}(u)+\operatorname{deg}^{+}(v) \geq 4$ for all but at most one $(u, v) \in E$, then $g \leq\lceil n / 2\rceil$. We also show for any digraph $G$ that, if $\operatorname{deg}^{+}(u)+\operatorname{deg}^{+}(v) \geq 3$ for all pairs of distinct vertices $u, v$ in $G$, then $g \leq\lceil n / 2\rceil$.

\section{Main Results}

Suppose $u$ is a vertex in $G$. If a digraph $G^{\prime}$ is obtained from $G$ by adding to it an extra vertex $u^{\prime}$ and $\operatorname{arcs}\left\{\left(u^{\prime}, v\right):(u, v) \in E\right\} \cup\left\{\left(v, u^{\prime}\right):(v, u) \in E\right\} \cup\left\{\left(u^{\prime}, u\right)\right\}$, we say that $G^{\prime}$ is obtained by using a copy transformation of $G$ at vertex $u$, and we call the vertex $u^{\prime}$ a copy vertex of $u$. It is straightforward, but a little tedious, to prove the following lemma. We only prove the third statement which is a refinement of the following observation: $g \leq d+1$ for all strongly connected digraphs.

Lemma 4 Suppose that $G^{\prime}$ is obtained by using a copy transformation of $G$ at vertex $u$ and that $u^{\prime}$ is the copy vertex of $u$. Then the following Statement 1 holds. Moreover, if $G$ is strongly connected, then all the following statements hold.

1. The girth $g\left(G^{\prime}\right)$ of $G^{\prime}$ equals the girth of $G$.

2. The diameter $d\left(G^{\prime}\right)$ of $G^{\prime}$ is at least the diameter of $G$.

3. $g\left(G^{\prime}\right) \leq d\left(G^{\prime}\right)$.

4. If $(v, u) \in E(G)$, then $\operatorname{deg}_{G^{\prime}}^{+}(v)=\operatorname{deg}_{G}^{+}(v)+1 \geq 2$. (Thus no arc starting from $v$ is a special arc in $G^{\prime}$ although it may be special in $G$.)

5. $\operatorname{deg}_{G^{\prime}}^{+}\left(u^{\prime}\right) \geq 2$. (Thus Statements 4 and 5 imply that no arc associated with $u^{\prime}$ is special in $G^{\prime}$.)

6. All special arcs in $G^{\prime}$ are also special in $G$. (Thus $G^{\prime}$ contains no more special arcs than $G$.)

Proof of Statement 3. Since $G$ is strongly connected, the construction of $G^{\prime}$ shows that $G^{\prime}$ is also strongly connected. Let $u \rightarrow u_{1} \rightarrow \cdots \rightarrow u_{l-1} \rightarrow u^{\prime}$ be a shortest path from $u$ to its copy vertex $u^{\prime}$ in $G^{\prime}$. By the construction of $G^{\prime}$, we have $\left(u_{l-1}, u\right) \in E(G)$ and so $\left(u_{l-1}, u\right) \in E\left(G^{\prime}\right)$. Thus $u$ lies on a cycle of length $l$ in $G^{\prime}$. Therefore $g\left(G^{\prime}\right) \leq l \leq d\left(G^{\prime}\right)$. 
Before stating the next theorem, we need some definitions. Suppose $u$ is a vertex in $G$. For any integer $i \geq 0$, let $D_{i}(u)$ (resp. $D_{i}^{\prime}(u)$ ) denote the set of vertices whose distance from (resp. to) $u$ is exactly $i$. In particular, $D_{0}(u)=D_{0}^{\prime}(u)=\{u\}$ since a vertex is at distance 0 from and to itself. Let $N_{i}(u)=\bigcup_{j=0}^{i} D_{j}(u)$, that is, $N_{i}(u)$ is the set of vertices whose distance from $u$ is at most $i$ in $G$.

Theorem 1 Suppose $G$ is strongly connected with order $n$, girth $g$ and diameter $d$. If $G$ contains no special arcs, then

$$
d \leq n-g
$$

Proof. Suppose Theorem 1 fails. Among all counterexamples with the minimum number of vertices, we choose $G$ to have the minimum number of vertices with outdegree exactly 1 . Let $u$ be a vertex such that $D_{d}(u) \neq \emptyset$. (This is possible by the definition of the diameter.) The following facts are occasionally used in the proof: $g \leq d+1$, $n=\sum_{j=0}^{d}\left|D_{j}(u)\right|$, and $D_{j}(u), 0 \leq j \leq d$, are pairwise disjoint non-empty sets. We make the following claims.

Claim 1: Suppose either $\left|D_{i-1}(u)\right|=1$ or $\left|D_{i}(u)\right|=1$ for some $i, 1 \leq i \leq g-1$. If

$$
\left|N_{i-1}(u)\right| \leq \begin{cases}2 g-3 & \text { if }\left|D_{i}(u)\right|=1, \\ 2 g-4 & \text { if }\left|D_{i}(u)\right| \geq 2,\left(\text { thus }\left|D_{i-1}(u)\right|=1,\right)\end{cases}
$$

then there are no $\operatorname{arcs}$ from $D_{i}(u)$ to $N_{i-1}(u) \backslash\{u\}$.

Proof of Claim 1: Otherwise, suppose $(v, w) \in E$ for some $v \in D_{i}(u)$ and some $w \in N_{i-1}(u) \backslash\{u\}$. Let $G_{1}$ be the subdigraph of $G$ induced by $N_{i-1}(u) \backslash\{u\}$. Let $G_{2}$ be obtained from $G_{1}$ by adding to it extra $\operatorname{arcs}(x, w)$ for all $x \in D_{i-1}(u) \cap D_{1}^{\prime}(v) \backslash D_{1}^{\prime}(w)$. Since either $\left|D_{i-1}(u)\right|=1$ or $\left|D_{i}(u)\right|=1$, it is easy to verify that, for all $y \in N_{i-1}(u) \backslash\{u\}$,

$$
\begin{aligned}
\operatorname{deg}_{G_{2}}^{+}(y) & = \begin{cases}\operatorname{deg}_{G}^{+}(y)-\left|D_{i}(u)\right| & \text { if } y \in D_{i-1}(u) \cap D_{1}^{\prime}(v) \cap D_{1}^{\prime}(w) \text { and }\left|D_{i}(u)\right| \geq 2, \\
& \text { (thus } \left.\left|D_{i-1}(u)\right|=1 \text { and } \operatorname{deg}_{G}^{+}(y) \geq\left|D_{i}(u)\right|+1,\right) \\
\operatorname{deg}_{G}^{+}(y)-\left|D_{i}(u)\right|+1 & \text { if } y \in D_{i-1}(u) \cap D_{1}^{\prime}(v) \backslash D_{1}^{\prime}(w) \text { and }\left|D_{i}(u)\right| \geq 2, \\
& \text { (thus } \left.\left|D_{i-1}(u)\right|=1 \text { and } \operatorname{deg}_{G}^{+}(y) \geq\left|D_{i}(u)\right|,\right) \\
\operatorname{deg}_{G}^{+}(y)-1 & \text { if } y \in D_{i-1}(u) \cap D_{1}^{\prime}(v) \cap D_{1}^{\prime}(w) \text { and }\left|D_{i}(u)\right|=1, \\
& \text { (thus } \left.\operatorname{deg}_{G}^{+}(y) \geq\left|D_{i}(u)\right|+1=2,\right) \\
\operatorname{deg}_{G}^{+}(y) & \text { otherwise }\end{cases} \\
\geq 1 &
\end{aligned}
$$

Thus $\delta^{+}\left(G_{2}\right) \geq 1$ and so it is easy to see that $g\left(G_{2}\right) \geq g-1$. Let $C$ be a strongly connected component of $G_{2}$ such that $C$ is also a sink of $G_{2}$; that is, there are no $\operatorname{arcs}$ from $C$ to $G_{2} \backslash C$ in $G_{2}$. (If $G_{2}$ itself is strongly connected, then $C=G_{2}$.) Let

$$
t=\left\{\begin{array}{lll}
0 & \text { if } & D_{i-1}(u) \cap D_{1}^{\prime}(v) \cap C=\emptyset, \\
1 & \text { if } & D_{i-1}(u) \cap D_{1}^{\prime}(v) \cap C \neq \emptyset \text { and }\left|D_{i}(u)\right|=1, \\
2 & \text { if } & D_{i-1}(u) \cap D_{1}^{\prime}(v) \cap C \neq \emptyset \text { and }\left|D_{i}(u)\right| \geq 2 .
\end{array}\right.
$$


Let $G_{3}$ be obtained by using $t$ copy transformations of $C$ at $w$. By Lemma 4 , for all $y \in C$,

$$
\operatorname{deg}_{G_{3}}^{+}(y) \geq \begin{cases}\operatorname{deg}_{G}^{+}(y)-\left|D_{i}(u)\right|+t \geq 3 & \text { if } y \in D_{i-1}(u) \cap D_{1}^{\prime}(v) \cap D_{1}^{\prime}(w), \\ & D_{i-1}(u) \cap D_{1}^{\prime}(v) \cap C \neq \emptyset \text { and }\left|D_{i}(u)\right| \geq 2, \\ \operatorname{deg}_{G}^{+}(y)-\left|D_{i}(u)\right|+t+1 \geq 3 & \text { if } y \in D_{i-1}(u) \cap D_{1}^{\prime}(v) \backslash D_{1}^{\prime}(w), \\ & D_{i-1}(u) \cap D_{1}^{\prime}(v) \cap C \neq \emptyset \text { and }\left|D_{i}(u)\right| \geq 2, \\ \operatorname{deg}_{G}^{+}(y) & \text { otherwise. }\end{cases}
$$

Also by Lemma 4, no arc associated with the copy vertices of $w$ is special in $G_{3}$ and $g(C)=g\left(G_{3}\right) \leq \begin{cases}d\left(G_{3}\right)+1 & \text { if } t=0 \\ d\left(G_{3}\right) & \text { if } t \geq 1\end{cases}$

Since $G$ contains no special arcs, $G_{3}$ contains no special arcs either. Since $n\left(G_{3}\right) \leq$ $\left|N_{i-1}(u) \backslash\{u\}\right|+t<\left|N_{i-1}(u)\right|+\left|D_{i}(u)\right|=\left|N_{i}(u)\right| \leq n$, by the choice of $G, G_{3}$ is not a counterexample to Lemma 1. Thus

$$
\begin{aligned}
d\left(G_{3}\right) & \leq n\left(G_{3}\right)-g\left(G_{3}\right) \leq\left|N_{i-1}(u) \backslash\{u\}\right|+t-g\left(G_{3}\right) \\
& \leq\left|N_{i-1}(u)\right|+t-1-g\left(G_{3}\right) \leq \begin{cases}2 g-4-g\left(G_{3}\right) & \text { if } t=0 \\
2 g-3-g\left(G_{3}\right) & \text { if } t \geq 1\end{cases}
\end{aligned}
$$

Since $d\left(G_{3}\right) \geq \begin{cases}g\left(G_{3}\right)-1 & \text { if } t=0 \\ g\left(G_{3}\right) & \text { if } t \geq 1\end{cases}$

we have $g\left(G_{3}\right) \leq g-2$, a contradiction to $g-1 \leq g\left(G_{2}\right) \leq g(C)=g\left(G_{3}\right)$. Therefore Claim 1 holds.

Claim 2: Suppose $\left|N_{i-1}(u)\right| \leq 2 g-5,\left|D_{i}(u)\right|=3$ and $\left|D_{i+1}(u)\right|=1$ for some $i$, $1 \leq i \leq g-2$. Let $D_{i+1}(u)=\{v\}$. If $\operatorname{deg}^{+}(v) \leq 2$, then $\left|D_{i-1}(u)\right| \geq 2$.

Proof of Claim 2: Otherwise suppose $g \geq 3$ and $\left|D_{i-1}(u)\right|=1$ for vertex $x$. Then, by Claim 1, there are no arcs from $D_{i}(u)$ to $N_{i-1}(u) \backslash\{u\}$. Also there are no arcs from $D_{i}(u)$ to $u$; otherwise $G$ would contains a cycle of length $i+1 \leq g-1$. Let $D_{i}(u)=\left\{w_{1}, w_{2}, w_{3}\right\}$. Suppose $\operatorname{deg}^{+}\left(w_{j}\right)=1$ for some $j, 1 \leq j \leq 3$. Then $\left(w_{j}, v\right) \notin E$ since $\operatorname{deg}^{+}(v) \leq 2$ and $G$ contains no special arcs. Thus $\left(w_{j}, w_{k}\right) \in E$ for some $k \neq j, 1 \leq k \leq 3$. Since $G$ contains no special arcs, $\operatorname{deg}^{+}\left(w_{k}\right) \geq 3$. Recall that $g \geq 3$ and that there are no arcs from $w_{k}$ to $N_{i-1}(u)$. Then $D_{1}\left(w_{k}\right)=\left\{v, w_{1}, w_{2}, w_{3}\right\} \backslash\left\{w_{k}\right\}$. Thus $\left(w_{k}, w_{j}\right) \in E$, which together with $\left(w_{j}, w_{k}\right) \in E$ imply $g=2$, a contradiction to $g \geq 3$. Therefore it may be supposed that $\operatorname{deg}^{+}\left(w_{j}\right) \geq 2$ for all $j, 1 \leq j \leq 3$. But this implies that, starting from each vertex in $D_{i}(u)$, there is an arc ends in $D_{i}(u)$ also. Thus $g \leq\left|D_{i}(u)\right|=3$, which implies $g=3$. By the choice of $G$, we have $d>n-g=\sum_{j=0}^{d}\left|D_{j}(u)\right|-3 \geq d+\left|D_{i}(u)\right|-3=d$, a contradiction, from which Claim 2 follows.

Claim 3: Suppose $\left|N_{i}(u)\right| \leq 2 g-3$ and $\left|D_{i+1}(u)\right|=1$ for some $i, 1 \leq i \leq g-2$. Let $D_{i+1}(u)=\{v\}$. If $\operatorname{deg}^{+}(v) \leq 2$, then $\left|D_{i}(u)\right| \geq 3$.

Proof of Claim 3: Otherwise suppose $g \geq 3$ and $\left|D_{i}(u)\right| \leq 2$. Let $X=N_{i}(u) \backslash\{u\}$. Then there are no arcs from $X$ to $u$; otherwise $G$ would contain a cycle of length at most 
$i+1 \leq g-1$. Let $G(X)$ be the subdigraph of $G$ induced by $X$. Then $\operatorname{deg}_{G(X)}^{+}(w) \geq 1$ for each vertex $w \in D_{i}(u)$; otherwise $(w, v)$ is the unique arc starting from $w$ in $G$ and so $(w, v)$ would be a special arc of $G$, a contradiction. Thus $\delta^{+}(G(X)) \geq 1$. For each $w \in D_{i}(u)$, let $w_{t}$ be a terminus of $w$ in $G(X)$; that is, $\left(w, w_{t}\right)$ is an arc in $G(X)$. Let $G_{1}$ be the digraph obtained by using a sequence of copy transformations of $G$ at each vertex in $\left\{w_{t}: w \in D_{i}(u)\right\}$. By Lemma $4, g\left(G_{1}\right)=g(G(X)) \geq g$. Since $G$ contains no special arcs, each possible special arc in $G(X)$ is associated with at least one vertex in $D_{i}(u)$. By Lemma 4 again, $G_{1}$ contains no special arcs. Let $C$ be a strongly connected component of $G_{1}$ such that $C$ is a sink of $G_{1}$. Then $C$ has order $n(C) \leq|X|+\left|D_{i}(u)\right|$ and girth $g(C) \geq g\left(G_{1}\right) \geq g$. Note that $n(C) \leq|X|+\left|D_{i}(u)\right| \leq \sum_{j=1}^{i}\left|D_{j}(u)\right|+2 \leq$ $\sum_{j=0}^{g-1}\left|D_{j}(u)\right| \leq \sum_{j=0}^{d}\left|D_{j}(u)\right| \leq n$. Suppose $n(C)=n$. Then $i+1=g-1=d$. This implies $\operatorname{deg}_{G}^{+}(v)=1$ since, by replacing $i$ by $i+1$ in Claim 1 , there are no arcs from $D_{i+1}(u)=\{v\}$ to $N_{i}(u) \backslash\{u\}=N_{d-1}(u) \backslash\{u\}=V \backslash\{u, v\}$ in $G$. Thus either $C$ has order less than $G$ or $C$ has fewer vertices with outdegree exactly 1 than $G$ does (since $v \notin C$ ). This implies that $C$ is not a counterexample to Lemma 1. Thus $d(C) \leq n(C)-g(C) \leq$ $|X|+\left|D_{i}(u)\right|-g(C) \leq\left|N_{i}(u)\right|+\left|D_{i}(u)\right|-1-g(C) \leq 2 g-2-g(C)$. Since $g(C)-1 \leq d(C)$, we have $g(C)-1 \leq d(C) \leq 2 g-2-g(C)$; i.e., $g(C) \leq g-1$, a contradiction to $g(C) \geq g$. Therefore Claim 3 follows.

Claim 4: Suppose $\left|N_{g}(u)\right| \leq 2 g-1$. Then either $\left|N_{i}(u)\right| \geq 2 i+1$ or $\left|N_{i+1}(u)\right| \geq 2 i+3$ for all $i, 0 \leq i \leq g-1$.

Proof of Claim 4: Claim 4 is trivial for $i=0$ and 1. Let $t$ be the first possible $i$, $2 \leq i \leq g-1$, satisfying $\left|N_{i}(u)\right| \leq 2 i$ and $\left|N_{i+1}(u)\right| \leq 2 i+2$. By the choice of $t$, we have $2 \leq t \leq g-1$ and $\left|N_{t-1}(u)\right| \geq 2 t-1$. Then $\left|D_{t}(u)\right|=1$; otherwise if $\left|D_{t}(u)\right| \geq 2$, then $\left|N_{t-1}(u)\right|=\left|N_{t}(u)\right|-\left|D_{t}(u)\right| \leq 2 t-2$, a contradiction. Thus $\left|N_{t-1}(u)\right|=2 t-1$ since $2 t-1 \leq\left|N_{t-1}(u)\right|=\left|N_{t}(u)\right|-\left|D_{t}(u)\right| \leq 2 t-1$. Also $\left|D_{t+1}(u)\right| \leq \begin{cases}2 & \text { if } 2 \leq t \leq g-2, \\ 1 & \text { if } t=g-1 ;\end{cases}$ otherwise $\left|N_{t+1}(u)\right|=\left|N_{t-1}(u)\right|+\left|D_{t}(u)\right|+\left|D_{t+1}(u)\right| \geq \begin{cases}2 t+3 & \text { if } 2 \leq t \leq g-2, \\ 2 g & \text { if } t=g-1,\end{cases}$ contradicting either the choice of $t$ or the assumption $\left|N_{g}(u)\right| \leq 2 g-1$. Let $D_{t}(u)=\{v\}$. Since $\left|N_{t-1}(u)\right|=2 t-1 \leq 2 g-3$, by setting $i=t$ in Claim 1, there are no arcs from $D_{t}(u)=\{v\}$ to $N_{t-1}(u) \backslash\{u\}$. Since there is no loop at $v$, we have $D_{1}(v) \subseteq \begin{cases}D_{t+1}(u) & \text { if } 2 \leq t \leq g-2, \\ D_{t+1}(u) \cup\{u\} & \text { if } t=g-1,\end{cases}$

Thus $\operatorname{deg}^{+}(v) \leq 2$ always holds. Since $\left|N_{t-1}(u)\right|=2 t-1 \leq 2 g-3$, by setting $i=t-1$ in Claim 3, $\left|D_{t-1}(u)\right| \geq 3$. Thus $\left|N_{t-2}(u)\right|=\left|N_{t-1}(u)\right|-\left|D_{t-1}(u)\right| \leq(2 t-1)-3=2 t-4$ and so $t \geq 3$. If either $\left|D_{t-1}(u)\right| \geq 4$ or $\left|D_{t-2}(u)\right| \geq 2$, then $\left|N_{t-3}(u)\right|=\left|N_{t-1}(u)\right|-$ $\left|D_{t-1}(u)\right|-\left|D_{t-2}(u)\right| \leq(2 t-1)-5=2 t-6$, which together with $\left|N_{t-2}(u)\right| \leq 2 t-4$ contradict the choice of $t$. Thus $\left|D_{t-1}(u)\right|=3$ and $\left|D_{t-2}(u)\right|=1$. On the other hand, since $\left|N_{t-2}(u)\right| \leq 2 t-4 \leq 2 g-6,\left|D_{t-1}(u)\right|=3,\left|D_{t}(u)\right|=1$ and $\operatorname{deg}^{+}(v) \leq 2$, by setting $i=t-1$ in Claim 2, we obtain $\left|D_{t-2}(u)\right| \geq 2$, a contradiction to $\left|D_{t-2}(u)\right|=1$. Therefore 
Claim 4 follows.

Claim 5: $d \leq n+i-\left|N_{i}(u)\right|$ for all $i \geq 0$. To justify this, since $n \geq\left|N_{i}(u)\right|$, it may be supposed that $i \leq d-1$. Therefore $n=\left|N_{i}(u)\right|+\sum_{j=i+1}^{d}\left|D_{j}(u)\right| \geq\left|N_{i}(u)\right|+d-i$, from which Claim 5 follows.

We are now ready to complete the proof. By setting $i=g-1$ in Claim 4 , we have either $\left|N_{g-1}(u)\right| \geq 2 g-1$ or $\left|N_{g}(u)\right| \geq 2 g$; i.e.,

$$
\min \left\{g-1-\left|N_{g-1}(u)\right|, g-\left|N_{g}(u)\right|\right\} \leq-g .
$$

By setting $i=g-1$ and $g$, respectively, in Claim 5,

$$
d \leq n+\min \left\{g-1-\left|N_{g-1}(u)\right|, g-\left|N_{g}(u)\right|\right\} \leq n-g .
$$

This completes the proof of Theorem 1 .

By the definition of the girth of a digraph, it is known that $d \geq g-1$ for any strongly connected digraph. The following construction shows that the bound $d \leq n-g$ in Theorem 1 is tight for the case $d \geq g$. Let $n=d+g$ and $d=g+s$, where $s \geq 0$. Let $V(G)=V_{1} \cup \ldots \cup V_{g} \cup\left\{u_{2 g+1}, \ldots, u_{n}\right\}$, where $V_{i}=\left\{u_{2 i-1}, u_{2 i}\right\}$ for all $1 \leq i \leq g$. Let $E(G)=\left(\cup_{i=1}^{g-1} E\left(V_{i}, V_{i+1}\right)\right) \cup\left(\cup_{i=2 g-1}^{n} E\left(u_{i}, V_{1}\right)\right) \cup\left\{\left(u_{i}, u_{i+1}\right): 2 g \leq i \leq n-1\right\}$, where $E\left(V_{i}, V_{i+1}\right)$ denotes the set of arcs from each vertex in $V_{i}$ to each vertex in $V_{i+1}$. Then $G$ has no special arcs. Also the girth of $G$ is $g$, and the diameter is $d$, which is achieved by the distance from $u_{2 g-1}$ to $u_{n}$.

If $(u, v)$ is an arc in $G$, we call $v$ the terminus of $(u, v)$. Since the number of special arcs is less than the number of vertices with outdegree exactly 1 in any digraph, the following Corollary 1 and Theorem 2 are improvement of [10, Theorem 1 and Theorem 2], respectively.

Corollary 1 Suppose $G$ is a strongly connected digraph of order $n$ with girth $g$ and diameter $d$. If $G$ contains $t$ special arcs, then $d \leq n-g+t$.

Proof. Let $u_{1}, u_{2}, \ldots, u_{s}$ be all termini of the special arcs in $G$. (A common terminus of more than one special arc counts only once.) Then $s \leq t$. Let $G^{\prime}$ be obtained by using a sequence of copy transformations of $G$ at each vertex in $\left\{u_{1}, u_{2}, \ldots, u_{s}\right\}$. Then $G^{\prime}$ is strongly connected of order $n+s$. By Lemma $4, G^{\prime}$ has girth $g$, diameter $d\left(G^{\prime}\right) \geq d$ and contains no special arcs. By applying Theorem 1 to $G^{\prime}, d \leq d\left(G^{\prime}\right) \leq n+s-g \leq n+t-g$.

Theorem 2 Suppose $G$ is a digraph of order $n$ with girth $g$ and $\delta^{+}(G) \geq 1$. If $G$ contains $t$ special arcs, then

$$
g \leq \begin{cases}\left\lceil\frac{n}{2}\right\rceil & \text { if } t=0 \\ \left\lceil\frac{n+t-1}{2}\right\rceil & \text { if } t \geq 1\end{cases}
$$


Proof. Without loss of generality, it may be supposed that $G$ is strongly connected; otherwise it suffices to consider a strongly connected component and also a sink of $G$. If $t=0$, then by Corollary $1, g-1 \leq d \leq n-g$; i.e., $g \leq\lceil n / 2\rceil$. Now suppose $t \geq 1$. Let $u$ be the terminus of a special arc and let $G^{\prime}$ be obtained by using a copy transformation of $G$ at $u$. Then $G^{\prime}$ is strongly connected of order $n+1$ with girth $g$ and $t-1$ special arcs. By Lemma 4 and Corollary $1, g=g\left(G^{\prime}\right) \leq d\left(G^{\prime}\right) \leq(n+1)-g+(t-1)=n-g+t$; i.e, $g \leq\lceil(n+t-1) / 2\rceil$.

Corollary 2 Suppose $G$ is a digraph of order $n$ with girth $g$ and $\delta^{+}(G) \geq 1$. If $G$ contains at most one special arc, then $g \leq\lceil n / 2\rceil$.

Proof. Since $t \leq 1$, Corollary 2 follows immediately from Theorem 2 .

The following corollary shows that any digraph $G$ with $\delta^{+}(G) \geq 1$ contains short cycles if the local average outdegree at each arc is at least 2.

Corollary 3 Suppose $G$ is a digraph of order $n$ with girth $g$ and $\delta^{+}(G) \geq 1$. If $\operatorname{deg}^{+}(u)+$ $\operatorname{deg}^{+}(v) \geq 4$ for all arcs $(u, v)$ in $G$, then $g \leq\lceil n / 2\rceil$.

Proof. Since $\operatorname{deg}^{+}(u)+\operatorname{deg}^{+}(v) \geq 4$ for all arcs $(u, v), G$ contains no special arcs. Therefore Corollary 3 follows from Corollary 2.

Remark 1. As shown by the following example, the condition $\delta^{+}(G) \geq 1$ in Corollary 3 cannot be dropped. Let $G=(V, E)$, where $V=A \cup B$ and $E=\{(u, v): u \in A$ and $v \in$ $B\}$, where $A \cap B \neq \emptyset,|B| \geq 4$ and $A$ is not empty. Then $\operatorname{deg}^{+}(u)+\operatorname{deg}^{+}(v) \geq 4$ for all $\operatorname{arcs}(u, v)$ in $G$. However $G$ does not even contain any cycles.

The following corollary shows that the condition $\delta^{+}(G) \geq 1$ may be dropped if $\operatorname{deg}^{+}(u)+\operatorname{deg}^{+}(v) \geq 3$ for all pairs of distinct vertices $u, v$ in $G$. Note that the latter condition implies that $G$ has at most one vertex $u$ such that $\operatorname{deg}^{+}(u) \leq 1$. Thus such a digraph $G$ contains at most one special arc.

Corollary 4 Suppose $G$ is a digraph of order $n \geq 2$ with girth $g$. If $\operatorname{deg}^{+}(u)+\operatorname{deg}^{+}(v) \geq 3$ for all pairs of distinct vertices $u, v$ in $G$, then $g \leq\lceil n / 2\rceil$.

Proof. By Lemma 1, it may be supposed that there is a unique $u$ such that $\operatorname{deg}^{+}(u) \leq$ 1. If $\operatorname{deg}^{+}(u)=1$, then $G$ contains at most one special arc and so Corollary 4 follows from Corollary 2. Now suppose $\operatorname{deg}^{+}(u)=0$. Then $\operatorname{deg}^{+}(v) \geq 3-\operatorname{deg}^{+}(u)=3$ for all vertices $v \neq u$. Let $G^{\prime}$ be the subdigraph of $G$ induced by $V \backslash\{u\}$. Then $G^{\prime}$ is a digraph of order $n-1$ with $\delta^{+}\left(G^{\prime}\right) \geq 2$. Thus by Lemma $1, g \leq g\left(G^{\prime}\right) \leq\lceil(n-1) / 2\rceil$, from which Corollary 4 follows. 


\section{Conclusion and Open Problem}

The Caccetta-Häggkvist Conjecture predicted a very interesting relationship among various fundamental parameters of digraphs: the girth, the degree and the number of vertices. It has been studied without general resolution since its appearance in 1978. Lacking appropriate methods to prove it, people tend to consider more general problems, in which the minimum outdegree condition $\delta^{+}(G) \geq r$ is relaxed so that it may be easier to employ induction and some other proof techniques. Given the above strategy, one could expect that the most difficult part is to find an appropriate 'generalized statement'. This has led to a number of stronger conjectures (see [3], for example). Motivated by Corollary 3, we present the following conjecture which is stronger than the Caccetta-Häggkvist Conjecture.

Conjecture 2 Suppose $G$ is a digraph of ordern with girth $g$ and $\delta^{+}(G) \geq 1$. If $\operatorname{deg}^{+}(u)+$ $\operatorname{deg}^{+}(v) \geq 2 r$ for all arcs $(u, v)$ in $G$, then $g \leq\lceil n / r\rceil$.

We conclude the paper with the following remarks:

Remark 2. By modifying the example in Remark 1 , we note that the condition $\delta^{+}(G) \geq 1$ in Conjecture 2 cannot be dropped.

Remark 3. The following family of digraphs has girth $\lceil n / r\rceil$ and satisfies the weaker condition that $\operatorname{deg}^{+}(u)+\operatorname{deg}^{+}(v) \geq 2 r$ for all arcs $(u, v)$ instead of the stronger one $\delta^{+}(G) \geq r$. Suppose $g$ is even and $1 \leq k \leq r-1$. Let $G=(V, E)$ with $V=\cup_{i=1}^{g} V_{i}$ and $E=\left\{(u, v): u \in V_{i}, v \in V_{i+1}, 1 \leq i \leq g\right\}$, where addition is taken modulo $g$. If $\left|V_{i}\right|= \begin{cases}r-k & \text { if } i \text { is odd, } \\ r+k & \text { if } i \text { is even, }\end{cases}$

then $G$ has girth $g$ and satisfies $\operatorname{deg}^{+}(u)+\operatorname{deg}^{+}(v)=2 r$ for all $\operatorname{arcs}(u, v)$. However $\delta^{+}(G)=r-k<r$. Therefore Conjecture 2 considers more digraphs than Conjecture 1 .

Remark 4. It is possible to apply the techniques presented in the paper to prove Conjecture 2 for some other lower values of $r$; however, the author feels that some other new techniques are needed in order to prove the general conjecture.

Acknowledgment I want to thank Professor Richard Brualdi and a referee for some helpful suggestions and comments.

\section{References}

[1] M. Behzad, Minimally 2-regular digraphs with given girth, J. Math. Soc. Japan 25 (1973), 1-6. 
[2] M. Behzad, G. Chartrand and C. Wall, On minimal regular digraphs with given girth, Fund. Math. 69 (1970), 227-231.

[3] J. A. Bondy, Counting subgraphs: A new approach to the Caccetta-Häggkvist conjecture, Discrete Math. 165/166 (1997), 71-80.

[4] L. Caccetta and R. Häggkvist, On minimal digraphs with given girth, Proc. 9th S-E Conf. Combinatorics, Graph Theory and Computing (1978), 181-187.

[5] V. Chvátal and E. Szemerédi, Short cycles in directed graphs, J. Combin. Theory, Ser. B 35 (1983), 323-327.

[6] Y. O. Hamidoune, A note on minimal directed graphs with given girth, J. Combin. Theory Ser. B 43 (1987), 343-348.

[7] C. T. Hoáng and B. Reed, A note on short cycles in digraphs, Discrete Math. 66 (1987), 103-107.

[8] T. Nishimura, Short cycles in digraphs, Discrete Math. 72 (1988), 295-298.

[9] J. Shen, Directed triangles in digraphs. J. Combin. Theory, Ser. B 74 (1998), 405-407.

[10] J. Shen, On the girth of digraphs. Discrete Math. 211(1-3) (2000), 167-181.

[11] J. Shen, On the Caccetta-Haggkvist conjecture, accepted by Graphs Combin. 18(3) (2002), 645-654. 